\title{
Manoeuvring Experiments Using the MUN Explorer AUV
}

\author{
Manoj T. Issac \\ $\mathrm{Ph} . \mathrm{D}$. Student, Ocean Engineering Research Centre (OERC) \\ Memorial University of Newfoundland (MUN), St. John's, NL, A1B 3X5, Canada \\ Sara Adams, Moqin He \\ Research Lab Coordinators, MERLIN \\ Memorial University of Newfoundland (MUN), St. John's, NL, A1B 3X5, Canada \\ Neil Bose \\ Professor, Canada Research Chair in Offshore and Underwater Vehicles \\ Memorial University of Newfoundland (MUN), St. John's, NL, A1B 3X5, Canada \\ Christopher D. Williams, Ralf Bachmayer
Research Engineer, National Research Council, Institute for Ocean Technology \\ St. John's, NL, A1B 3T5, Canada
}

\begin{abstract}
Autonomous Underwater Vehicles (AUVs) are self-propelled robotic platforms that can perform a predetermined mission completely unmanned. A series of manoeuvring experiments were performed using the $M U N$ Explorer AUV during the summer of 2006 and a selection of these experimental results are presented in this paper. The purpose of these experiments was to collect a set of useful data for validating a hydrodynamic model of the dynamic performance of the vehicle. This paper aims at explaining the methods and measures adopted in accomplishing this task. Apart from providing a data set for validation of the hydrodynamic model, the data record also shows the ability of the AUV to perform extreme manoeuvres and the accuracy with which it can follow a pre-planned mission.
\end{abstract}

\section{INTRODUCTION}

Autonomous Underwater Vehicles (AUVs) are rapidly emerging as an important tool in ocean exploration and monitoring. A number of oceanographic problems require quality data acquisition without disturbing the ocean environment. Ship-borne methods can introduce errors in measurements due to the disturbance caused by towed instrument packages, samplers or by the ship itself. The potential of AUV technology in undersea surveys and the significant improvement in the quality of data acquired using these platforms, is gradually being recognised as a replacement for conventional ship-borne methods.

During a mission, an AUV may undergo different manoeuvring scenarios such as a complete turn at the end of a survey line, a severe turn during obstacle avoidance or frequent depth changes while following a rugged seabed terrain. This demands a higher degree of manoeuvrability in order to achieve good position and attitude control. The designers of AUVs increasingly rely on computer models (hydrodynamic models) to study the motion behaviours and in turn to develop vehicle controllers.

Hydrodynamic models are often developed to predict the performance of these vehicles, especially their interaction with the fluid. A reliable hydrodynamic model is one that can closely predict or reproduce the actual vehicle operational scenarios. A dynamics model of an AUV based on the "body-build-up" method of Nahon [1] was developed by Perrault [2] and Evans [3] using MATLAB and SIMULINK and is available for use at Memorial University. To validate the model, it is necessary to have experimental data from a real vehicle. As a result, a series of open-water manoeuvring tests was completed using the MUN Explorer AUV during the summer of 2006.

The MUN Explorer is a survey-class AUV recently acquired by Memorial University of Newfoundland, in June 2006, from International Submarine Engineering Limited (ISE) in Port Coquitlam, British Columbia. It was funded by the Atlantic Canada Opportunities Agency through the Atlantic Innovation Fund. Offshore environmental monitoring and seabed surveys are some of the primary areas of research for which the vehicle will be utilized. The vehicle is made available for graduate students' research.

This paper describes the methods and measures taken to accomplish a series of manoeuvring tests using the MUN Explorer AUV. Results from a selected number of experiments are presented in this paper along with the lessons learned.

\section{THE MUN EXPLORER AUV}

The MUN Explorer is a survey-class AUV and functions mainly as a sensor platform for undersea data collection purposes. The AUV is $4.5 \mathrm{~m}$ in length with a maximum diameter of $0.69 \mathrm{~m}$ and is designed to go as deep as $3000 \mathrm{~m}$. It is propelled by a twin-bladed propeller and can reach cruising speeds between 0.5 to $2.5 \mathrm{~m} / \mathrm{s}$. Six control-planes assist with manoeuvring of the vehicle. To the aft of the vehicle is a retractable communications mast that can be raised to approximately $1.0 \mathrm{~m}$ above the body of the vehicle. The vehicle has a dry weight of $630 \mathrm{~kg}$. 


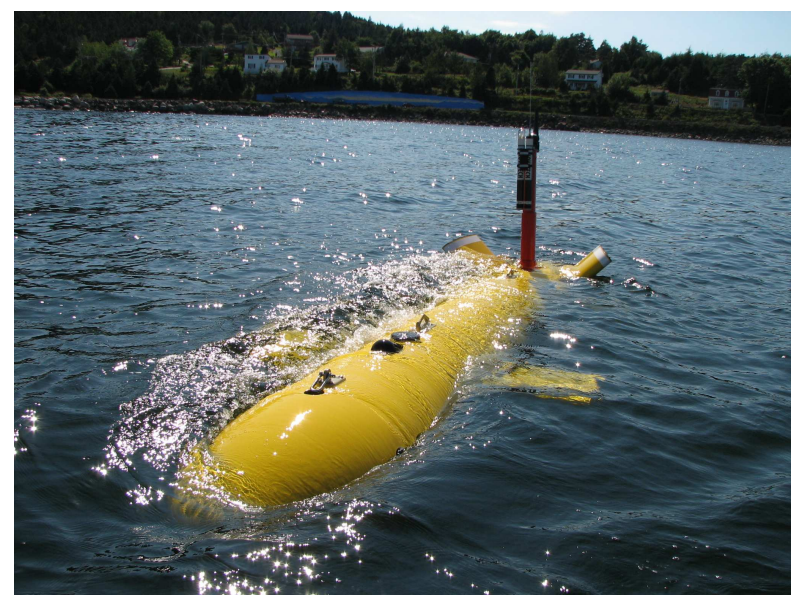

Fig. 1 The MUN Explorer AUV

An onboard computer controls the motion of the AUV and communicates periodically with the surface console computers. This vehicle can carry approximately $150 \mathrm{~kg}$ of scientific payload without having to add extra buoyancy in the form of syntactic foam. Fig. 1 shows a picture of the MUN Explorer AUV on the surface after a mission.

\section{A. Structure}

The MUN Explorer AUV is modular in structure consisting of a cylindrical main body blended with a nose cone at its front and a tapered tail section at its rear, giving it a hydrodynamically efficient streamlined shape. Except for the pressure hull, the majority of the hull section is made of Glass Reinforced Plastic (GRP) thus greatly reducing the overall weight of the vehicle. The pressure hull consists of a ring stiffened cylindrical module made of cast 7075-T6 Aluminium alloy with two 7075-T6 Aluminium alloy hemispherical end caps. The end-caps are joined to the cylinder using split aluminium clamps. The pressure hull provides the majority of the buoyancy for the vehicle and space for dry components such as batteries and control electronics.

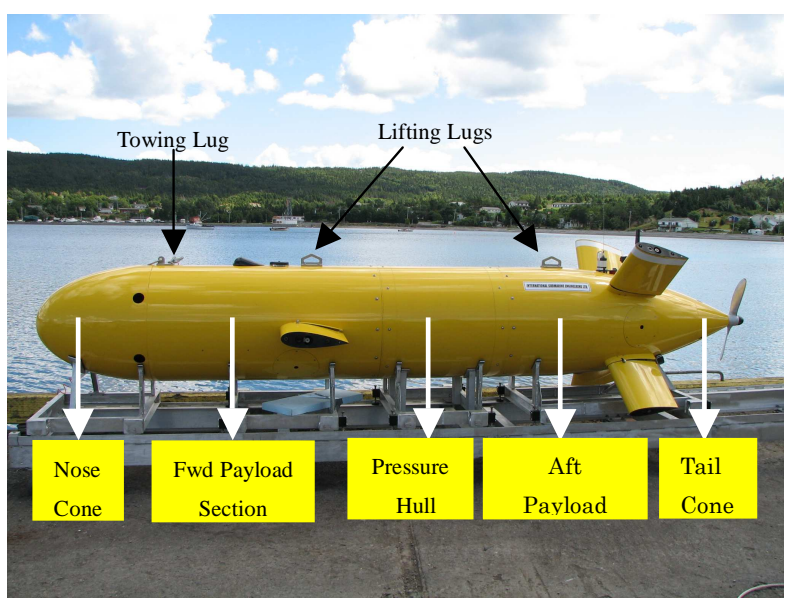

Fig. 2 Modular Sections of the AUV

Immediately forward of the pressure hull is the free-flooding forward payload section. This section has the forward planes, forward lifting lug, towing lug, depth sensor, LinkQuest ${ }^{\mathrm{TM}}$ acoustic transponder and the ORE acoustic transponder mounted in it. Space is available inside this section for additional wet payload items.

The free-flooding aft payload section made of GRP is to the rear of the pressure hull and it has a tapered end to connect to the tail cone section. Some navigation sensors and devices, such as the Doppler Velocity Log (DVL) and retractable communication mast are located in this section. There is room for some additional wet payload in this section. The aft control planes are also attached to this section. The tail cone is like a torpedo tail, and is designed to reduce the drag caused by the pressure drop at the end of the vehicle body. The propeller, drive motor and gearbox are located in this section. Fig. 2 shows the modular sections of the AUV.

\section{B. Control and Guidance}

The Vehicle Control Computer (VCC) is the "brain" of the vehicle providing guidance and control using both AUV sensors and actuators. It acquires data from all onboard instruments and sends these data to the Surface Control Console (SCC). It also receives commands from the surface or onboard control systems and generates commands for its actuators.

A network of computers on the surface provide AUV mission planning, monitoring and access to certain positioning sensors. It transmits all pilot commands to the vehicle and generates graphics text and diagnostics displays to provide information to the operator through a custom designed graphic user interface (GUI).

\section{Communication Systems}

Although an AUV is capable of autonomous operations, it is often advantageous to maintain a contact with the vehicle from the surface. This enables the operator to have occasional health checks and also ensures that the AUV sensors are logging useful information. The communication between the AUV (VCC) and the SCC is established by three different techniques:

\section{i) Data Radio Telemetry Link}

The radio telemetry system is capable of high-speed communications with the on-shore console while the AUV is on the surface. For this communication, two wireless Ethernet Datalinc SRM6210E $900 \mathrm{MHz}$ radio modems are used; one is installed in the vehicle and the other is on the on-shore console. The radio link can be used for piloting the AUV and for uploading missions to the vehicle.

Radio contact is possible only at the start and completion of a mission when the AUV is on the surface. The acoustic system is not functional at the surface because the top-mounted transponder is out of the water. At the end of a mission, the radio link is used to pilot the AUV to a safe recovery position and shut it down.

\section{ii) Underwater Acoustic Telemetry}

The underwater acoustic system consists of the LinkQuest, electronic transducer which is mounted in the forward payload section. The LinkQuest system is used to transmit data between the AUV and the surface when the AUV is underwater. It can be enabled from the SCC-GUI 
by the operator.

iii) Hardwire Deck Cable

A high-speed hardwired $100 \mathrm{Mb}$ Ethernet link is used for working with the vehicle on the deck (wharf). This link is used for uploading missions, retrieving data after a mission, as well as for normal pre and post-dive checks.

\section{Navigation and Positioning}

The navigation system of the vehicle consists of several different sensors to locate and orient the vehicle in 3-D space. A pressure sensor (Paroscientific - RS232 $200 \mathrm{~Hz}$ ) located in the forward payload section measures the depth of vehicle below the water surface, while bottom avoidance and altitude are provided using a forward looking altimeter [Kongsberg Acoustic Altimeter (KSM1007)] located in the nose of the vehicle. The geographical coordinates of the position are obtained by means of a RDI velocity sensor (RDI Workhorse $300 \mathrm{kHz}$ DVL) when the vehicle is submerged. The DVL is an Acoustic Doppler Current Profiler (ADCP), which has four acoustic beams that measure velocity parallel to the four beams. The VCC uses this velocity and heading data to correct the position from the inertial navigation system. However, it needs to be initialized or reset intermittently by position fixes when at the surface from a GPS. The MUN Explorer uses a Sound Ocean Systems DGPS, mounted on the retractable communications mast that determines precise positions (geographical coordinates) while the AUV is on the surface.

\section{E. Propulsion and Manoeuvring}

The AUV is propelled by a $0.65 \mathrm{~m}$ diameter high efficiency twin-bladed propeller driven by a Hathaway 48 VDC brushless motor and is capable of achieving a maximum speed of $2.5 \mathrm{~m} / \mathrm{s}$. The propeller is blended into the tail cone to maintain attached flow for better hydrodynamics.

Manoeuvring of the vehicle in 3-D space is facilitated by six control planes - four aft planes arranged in an " $\mathrm{X}$ " configuration and two foreplanes which assist with precise depth and roll control. The vehicle yaw, pitch and roll motions can be independently controlled by the aft planes. With proper control of the vehicle pitch, the vehicle depth can also be controlled using only the aft planes. The optional twin foreplanes are not always needed, but they can be useful if there is a need for independent depth and pitch control as, for e.g., in a precision terrain-following operation. The planes have a symmetrical cross-section (NACA 0024). Each plane is controlled independently by a 24 Volt brushless DC motor that resides inside the plane body.

\section{F. Energy System}

The primary source of energy is a bank of E-One Moli Energy $1.1 \mathrm{kWh}$ Lithium ion battery modules. Each module is furnished with a cell monitoring and charging system to manage its output. There is room for 12 such modules and these are housed inside the pressure hull. At the time of test, the MUN Explorer used two sets of batteries while the remaining vacant space was occupied by lead ballast of equivalent weight. The vehicle operates on a bus voltage of 48 VDC.

\section{G. Emergency Systems}

The vehicle is equipped with a xenon flasher (Novatech ST-400-AR) and a radio beacon (Novatech RF-700A1) built by Novatech Design Ltd., as emergency devices. These are independently powered by its batteries. The strobe-light automatically turns itself on when the illumination is poor or at night and indicates to the surrounding area the presence of the AUV. The radio beacon is also self-powered and automatically turns itself on when the vehicle is on the surface, so that the vehicle can be found by using the corresponding radio direction finder when within range. The GPS antenna, radio telemetry antenna, RF beacon and strobe light are all mounted on top of the retractable communications mast. On diving, the mast automatically retracts into the hull and remains flush to the hull. When radio communication is needed or a GPS position fix is necessary, the vehicle can climb near the surface at low speed and the mast can be extended approximately $1.0 \mathrm{~m}$ above the vehicle body to reach out of the water as seen in Fig. 1. It can also be triggered from the SCC while the AUV is on the surface.

To locate the AUV when it is running underwater, an acoustic device must be relied upon. MUN Explorer uses a self-powered ORE LXT 4336 vehicle transducer as an emergency beacon.

More details about the vehicle can be found in reference [4]. The Explorer class vehicle has been used for environmental monitoring missions by IFREMER [5]. Eagle Ray is another Explorer class AUV owned by the National Institute for Undersea Science and Technology at the University of Southern Mississippi [6]. The National Undersea Research Centre at the University of North Carolina, Wilmington, has also operated the vehicle for high-resolution habitat mapping of deep offshore areas [7].

\section{EXPERIMENTAL SET-UP}

The tests reported here using the MUN Explorer AUV were performed during the month of August 2006. The entire test was planned to be carried out at Holyrood Harbour situated 28 miles South-West of St. John's, Newfoundland. This sheltered body of water had the requisite depth and spread along with onshore facilities, to carry out all the intended tests. The water depth ranged from 10 to $50 \mathrm{~m}$ or more in a span of 1 to $2 \mathrm{~km}$. A topographical map of the location as seen in the "FleetManager" software is shown in Fig. 3. AUVs are often launched from a support vessel. However, due to favourable site conditions and limited scope of the test, the deployment and recovery of the vehicle was done from the wharf using a crane truck. Once the vehicle was launched, it was towed to a safe distance away from the wharf before any missions were executed. 


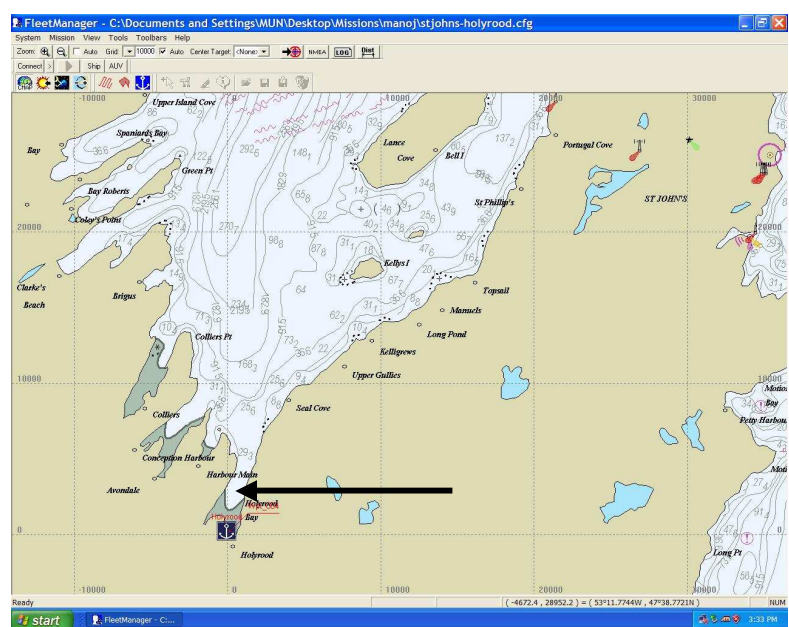

Fig. 3 Topography of the location - Holyrood as seen in "FleetManager"

The operations control station was housed in a container located on the wharf consisting of two PCs in a network: SCC and the other is used as a mission planning work station. The operator console displays vehicle information in graphical and text forms and also accepts commands for actuators and instruments. The operator console runs on a QNX 4.25 Operating System. The mission planning console runs the graphical chart display and mission supervision software "FleetManager". It aids in preparing the mission plans and is run on a Windows XP platform.

The "FleetManager", software was developed by the company Advanced Concept and System Architecture (ACSA), France [8] for intelligent mission preparation, and way-point generation and is dedicated to supervision of multiple vehicles. The system is based on C-Map software.

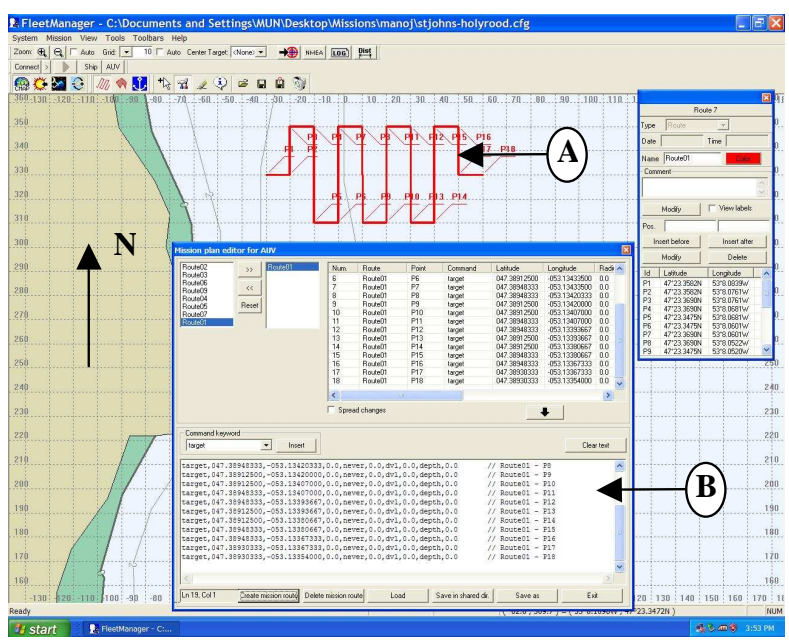

Fig. 4 Sample FleetManager Display of a Mission Plan

AUV missions are defined as ASCII text files. The FleetManager contains tools and features allowing you to create missions with both drawing tools and a text editor that understands the AUV mission file syntax. It contains mission task verbs, built-in keywords and comments. For instance, entry label and goto are two built-in keywords which are used for looping and jumping within a mission. The task verbs fall into two categories: geographic tasks and other. The geographic tasks are related to a geographical position (latitude and longitude) and are generated graphically. The list of geographic task verbs includes target, line_follow, circle and circle_current. Each geographic task has a configurable vertical mode (depth or altitude); a vertical setpoint, a speed mode and a speed setpoint. The other types of tasks include the ability to turn equipments on or off for a specific part of a mission.

FleetManager permits the user to create manual and automatic routes. By using a series of geographic task verbs, a manual route can be defined as a series of points as denoted by "A" in Fig. 4. By differing depth or altitude setpoints at each point, it is possible to create virtually any trajectory in 3D-space. Later, the built-in editor, denoted by "B" in Fig. 4, can be used to improve the routes, specific geographic parameters such as vertical mode, speed etc. The mission files after creation are saved with the extension '*.mis'. Since the mission plans are simple text files, any text editor can be used to create and modify them.

The mission plan file generated using the mission planning console is later transferred to the operator console which in turn is downloaded to the VCC through one of the data links. To overcome the problem of loading the missions one at a time, several missions consisting of similar manoeuvres were gathered together and compiled into a single large mission file with each mission demarcated by a unique label number (entry_label). The missions were also designed in such a way that after the completion of each mission (manoeuvre) the vehicle comes to the surface before starting the next mission. This informed the operator on the surface of the completion of a manoeuvre. The data from all instruments were logged on to the VCC during the entire operating period. A sampling frequency of $10 \mathrm{~Hz}$ was chosen for the entire test. After recovery of the vehicle, all data log files were uploaded to the SCC for further analysis.

The following section describes the different manoeuvring tests performed using the vehicle. It also presents data from a few examples.

\section{MANOEUVRING}

Manoeuvring trials are often done with free-running models to assess the stability and controllability of the vehicle. The purpose of the test here was to obtain a set of data from a real vehicle for validation of a hydrodynamic motion simulation model. Hence a series of standard manoeuvres were planned. These included straight-line test (acceleration/deceleration), horizontal and vertical zigzags, turning circles and helices. In order to avoid the effect of any surface disturbances, all horizontal plane manoeuvres were performed at a depth of approximately 3.0 metres. For manoeuvres in the vertical plane, the vehicle was commanded to dive even deeper such that the entire mission was done in a band with depth ranging between 3 
to $12 \mathrm{~m}$. In order to excite all 6 degrees-of-freedom of the vehicle a helix manoeuvre was also done.

\section{A. Acceleration/Deceleration Test}

An acceleration test is performed by increasing the speed of the vehicle from rest or from a particular ahead speed to a higher ahead speed while deceleration is by decreasing it from a speed to a lower speed or until the vehicle comes to a rest [9]. The test was performed at a depth of approximately 3.0 metres. Starting the vehicle from rest or bringing the vehicle to a complete stop was not possible with the underwater vehicle at this depth. To make the vehicle dive to a depth of 3.0 metres, the vehicle has to be moving at a considerable speed such that the control planes are effective in making it dive. An AUV is often designed to be slightly positively buoyant. For this reason, it was difficult or impossible to keep the vehicle at rest at any depth except at the surface, because the vehicle slowly rises to the surface. Also with MUN Explorer the control system architecture does not permit the propeller rpm to be set to zero as this indicates a fault condition.

To overcome these above difficulties, it was decided to do the test by changing the speed from a lower to higher value for acceleration and vice-versa for deceleration while the vehicle followed a straight-line path at a depth of 3.0 metres. Fig. 5 shows the plot of velocity variation during the acceleration and deceleration test. To make it dive to 3.0 metres, the vehicle was programmed to start from the surface at a speed of $1.5 \mathrm{~m} / \mathrm{s}$. Once the vehicle had levelled off at that depth, it was programmed to change to different speeds. From Fig. 5, it can be seen that the vehicle decelerates from a maximum speed of $2.5 \mathrm{~m} / \mathrm{s}$ to as low as $1.0 \mathrm{~m} / \mathrm{s}$ in steps of $0.5 \mathrm{~m} / \mathrm{s}$. The heading was towards North. Each speed was maintained for a length-of-travel of $100 \mathrm{~m}$. At the end of the straight-line route it turned back to the South and accelerated from a speed of $1.0 \mathrm{~m} / \mathrm{s}$ to $2.5 \mathrm{~m} / \mathrm{s}$ in steps of $0.5 \mathrm{~m} / \mathrm{s}$. A total of two such manoeuvres were performed.

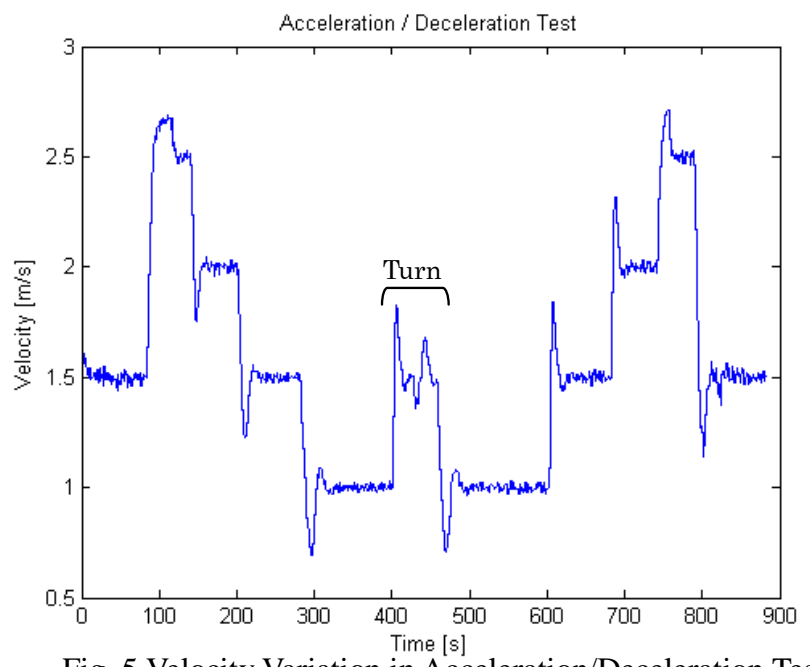

Fig. 5 Velocity Variation in Acceleration/Deceleration Test

\section{B. Horizontal and Vertical Zigzags}

Zigzag manoeuvres are indicative of the controllability of the vehicle. They show the effectiveness of the rudders in controlling the vehicle. A zigzag manoeuvre is performed by deflecting the rudder to a pre-defined angle and holding it until the vehicle heading has changed to the same angle. Then the rudder is deflected to the same angle in the opposite direction and held in place until the vehicle heading changes to that value, which completes a cycle. This procedure is followed for three or four cycles. The rudder angle and the forward speed are the control inputs. However, this conventional method was not possible because the vehicle control software architecture does not permit one to control the rudder directly. Hence an alternative method had to be found.

The mission routes were planned using the geographic task verb target thereby picking grid-points on the electronic chart. As mentioned the missions were planned in the North-South direction where the vehicle was set to do a horizontal zigzag on the way North and then turned at the end of the mission and performed a vertical zigzag on the way South. This saved a lot of time and energy. One such mission route is shown in Fig. 6.

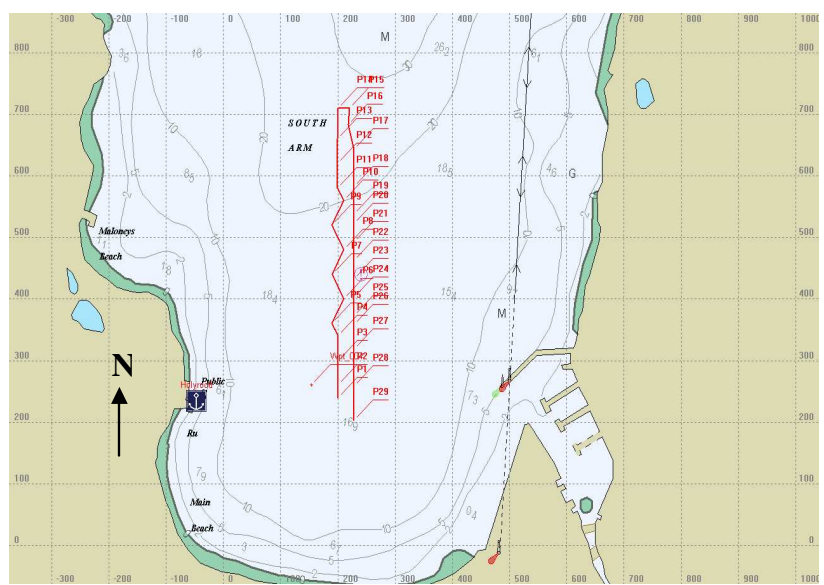

Fig. 6 Mission Plan for a Horizontal \& Vertical Zigzag

The zigzag missions were gathered together into a single large mission file with the horizontal and the vertical mission plans alternating with each other. This ensured that the vehicle always did a horizontal zigzag on its way up and a vertical zigzag on its way down. As mentioned in Section III, virtually any 3-D trajectory can be made by defining the position coordinates of a point on the chart. The vehicle was made to follow a zigzag in the vertical plane by changing the vertical mode information (depth) of each point on the chart while this was kept constant in the case of a horizontal zigzag. The following plot shows the result from a horizontal zigzag. 


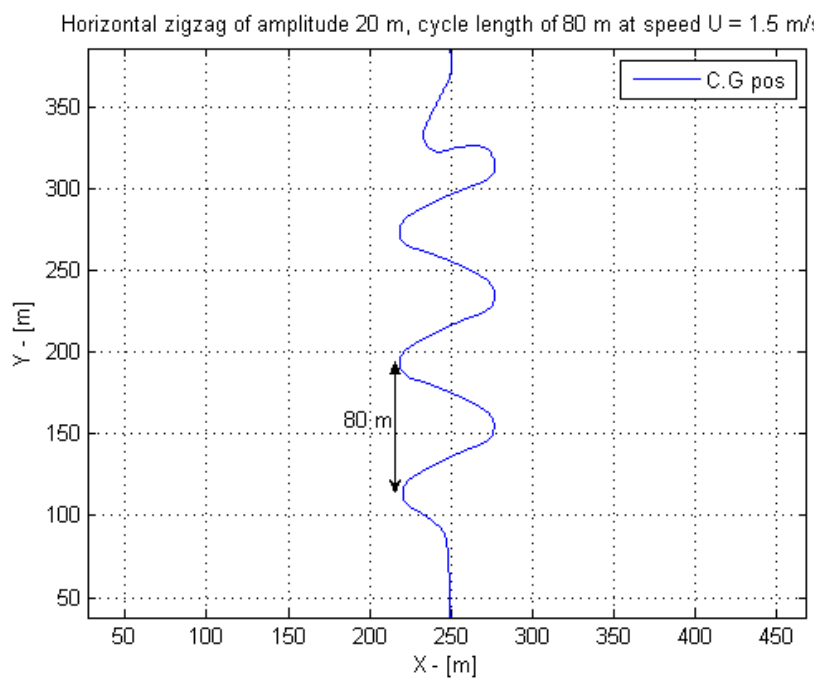

Fig. 7 Horizontal zigzag at forward speed, $U=1.5 \mathrm{~m} / \mathrm{s}$

The commanded amplitude and cycle-length for the zigzag were $20 \mathrm{~m}$ and $80 \mathrm{~m}$ respectively. From a preliminary analysis, it was found that there is an overshoot of approximately $8 \mathrm{~m}$ in the amplitude value while the cycle-length was maintained at $80 \mathrm{~m}$. A detailed analysis of the data will be presented in a future publication. A total of six horizontal manoeuvres were completed during the test period.

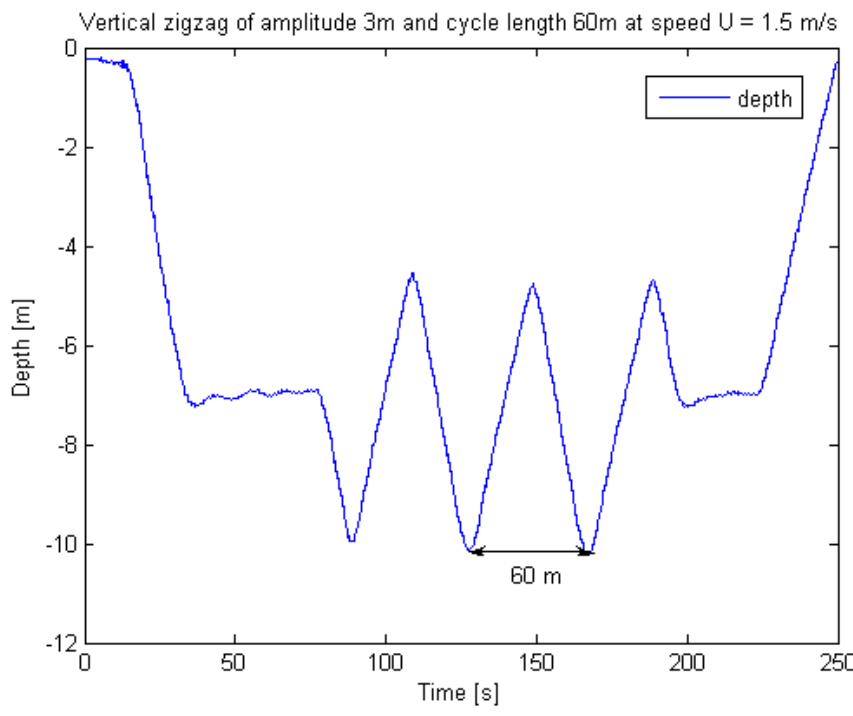

Fig. 8 Vertical zigzag at forward speed, $U=1.5 \mathrm{~m} / \mathrm{s}$

The depth profile from a vertical zigzag is shown in Fig. 8. The mission commanded the vehicle to dive to a mean depth of around 7 metres, which in turn is the reference level for the vertical zigzag shown. By commanding the vehicle to perform a vertical zigzag of amplitude $3 \mathrm{~m}$, the entire mission was completed in a band of depth ranging from $4 \mathrm{~m}$ to $10 \mathrm{~m}$. This could be considered as an extreme manoeuvre as the vehicle had to climb or dive $6 \mathrm{~m}$ in a span of $30 \mathrm{~m}$. It was found that there was hardly any overshoot in this manoeuvre. This was primarily because the vehicle used the foreplanes to dive and climb rather than the tail planes. A total of six manoeuvres were performed in the vertical plane.

\section{Turning Circles}

The turning circle manoeuvre is a steady-state manoeuvre in which the vehicle enters a steady turn at a constant speed. It is performed by deflecting the rudders to a predefined angle and holding it still resulting in the vehicle entering a circular path. The turning radius and rate of turn are characteristics of a particular turning path. Again, in the classic manoeuvre the control inputs are normally rudder angle and forward speed.

The vehicle controller, in the case of MUN Explorer, does not permit the user to set rudder deflection angles directly; instead, it allows the user to set a radius of turn. Hence the control inputs here were radius of turn and forward speed, contrary to rudder angle and forward speed in the conventional case. The task verb circle was used to generate all the turning circle missions and was performed at a depth of $3.0 \mathrm{~m}$. A total of 18 turning circles of various turning radii at different speeds were completed during the test period. Fig. 9 shows a sample result from a typical turning manoeuvre.

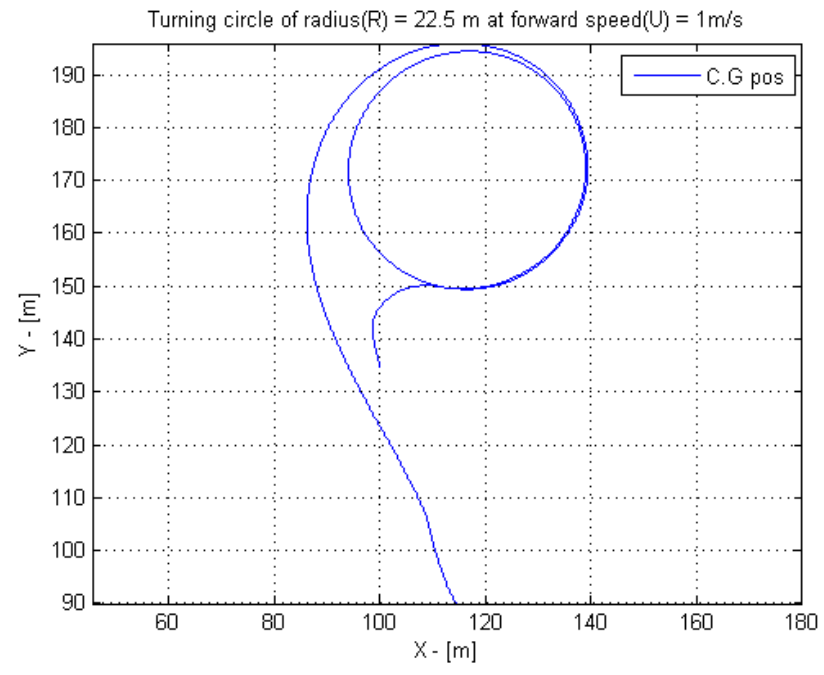

Fig.9 Turning circle at forward speed, $U=1.0 \mathrm{~m} / \mathrm{s}$

\section{Helix Manoeuvre}

The manoeuvres described in the above subsections are all planar manoeuvres; that is, the vehicle moves either in a horizontal or vertical plane only. This need not necessarily excite all degrees-of-freedom (DOF) of motion. The helix manoeuvre is not a standard manoeuvre but was designed to excite all six degrees-of-freedom. A 3D motion in which the vehicle spiralled down in circles was considered to excite all 6 DOF. Also this seemed well within the capability of the vehicle controller. As a result, the mission was planned using a series of geographic task verbs circle by changing the depth information of each circle commands. Fig. 10 shows the 3D plot of the helical manoeuvre performed by the vehicle. 


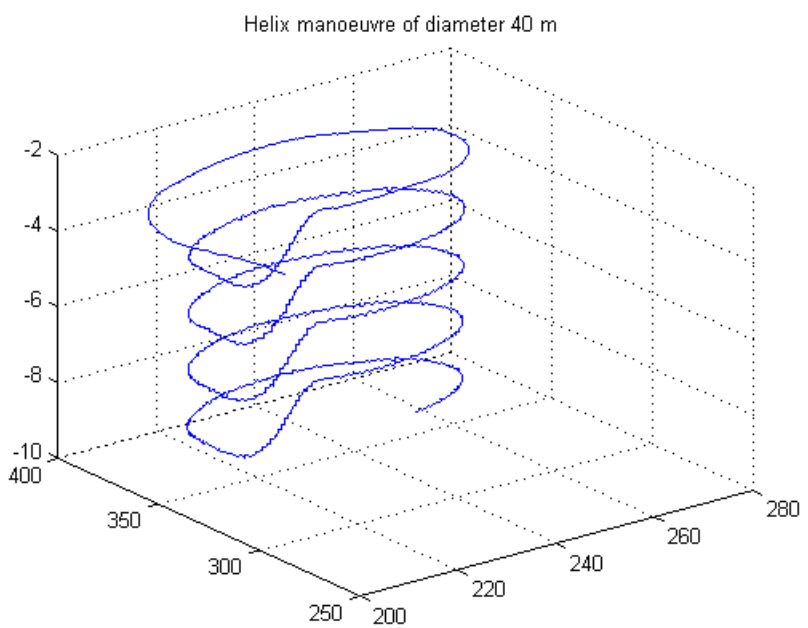

Fig.10 3D plot of the helical manoeuvre

The vehicle was planned to go in circles of radius $20 \mathrm{~m}$ while diving simultaneously such that it reached exactly $1.5 \mathrm{~m}$ below the starting point at the end of a circle. In other words, the vehicle was expected to dive continuously as it went around in circles. However, this was not the case as can be seen from the figure. The vehicle seemed to have completed almost a circle at each level and then dropped to the next level, completed almost another circle and so on.

\section{CONCLUSIONS}

A series of manoeuvring tests using the MUN Explorer AUV was completed in the summer of 2006. Sample results from a few of these manoeuvres are presented in this paper. This paper also describes the methods and procedures used in accomplishing the task along with the difficulties and lessons learned. A total of 18 turning circles, 12 zigzags, 2 straight-line tests and a helix were done during the test period. All manoeuvres were done in a slightly different manner compared with the classic maneuvers in order to make use of the vehicle mission planning software From a preliminary analysis of the zigzag manoeuvres, the vehicle seems to have a good path-follow qualities. In the case of turning circles, the radius of turn in most cases turned out to be somewhat more than the demanded radius. Detailed analysis of the entire data set will be presented in a future publication. Apart from providing a data set for validation purpose, the data records also show the ability of the vehicle to perform extreme manoeuvres and the accuracy with which it can follow a pre-defined path.

\section{ACKNOWLEDGEMENTS}

The authors wish to thank graduate students Haibo Niu and Jihad Shaana for their support and help in accomplishing this task.

\section{REFERENCES}

[1] M. Nahon, 1996 "A Simplified Dynamics Model for Autonomous Underwater Vehicles", Autonomous Underwater Vehicle Technology, AUV '96, pp. 373 379.

[2] D.E. Perrault, 2002 "Autonomous Underwater Vehicles (AUV) Sensitivity of Motion Response to Geometric and Hydrodynamic parameters and AUV Behaviours with Control Plane Faults", Ph.D. thesis, Memorial University of Newfoundland, February 2002.

[3] J. Evans and M. Nahon, 2004 "Dynamic modeling and performance evaluation of an autonomous underwater vehicle”, Ocean Engineering, 31, 1835 - 1858.

[4] EXPLORER, ISE Web Based AUV DesignInfo, International Submarine Engineering Ltd. (www.ise.bc.ca - accessed on January, 2007)

[5] V. Rigaud, J. Louis, J.S. Ferguson, J.M. Laframboise, T. Crees, P.Leon, J. Opderbecke, Y. Chardard, "First Steps in Ifremer's Autonomous Underwater Vehicle Program- A 3000m Depth Operational Survey AUV for Environmental Monitoring", Proceedings of the Fourteenth International Offshore and Polar Engineering Conference, Toulon, France, May 23 - 28, 2004.

[6] http://www.usm.edu/niust/uvtchome3.htm

[7] http://www.uncw.edu/nurc/auv/oculina2006/summary/

[8] A.C.S.AUnderwater GPS, http://www.underwater-gps.com

[9] E. V. Lewis, Principles of Naval Architecture, Vol. III, pp $251-256$. 
